\title{
A Novel Structure for MMSE Transceivers over Slowly Time-varying Channels
}

\author{
Chih-Hao Liu and P. P. Vaidyanathan \\ Dept. of Electrical Engineering, MC 136-93 \\ California Institute of Technology, Pasadena, CA 91125, USA \\ E-mail: chliu@caltech.edu and ppvnath@systems.caltech.edu
}

\begin{abstract}
This paper addresses the design problem of decision feedback (DF) transceiver without zero-forcing constraint over slowly time-varying narrowband multi-input multi-output (MIMO) channels. The space-time generalized triangular decomposition (ST-GTD) is applied for the design of minimum mean square error (MMSE) DF transceiver. With space-time powerloading, the proposed space-time geometric mean decomposition (ST-GMD) MMSE transceiver maximizes Gaussian mutual information over the equivalent channel seen by each space-time block. For practical applications, the causal ST-GTD MMSE transceiver which does not require channel prediction but shares the same asymptotic bit error rate (BER) performance with the ST-GMD MMSE system is also developed. In high signal to interference plus noise ratio (SINR) region, our results show that the proposed MMSE transceivers have better BER performance than the conventional GMD-based MMSE transceiver; the average BERs of the proposed systems are a non-increasing function of the ST-block size. ${ }^{1}$
\end{abstract}

Index Terms-Generalized Triangular Decomposition, Transceivers, GMD, Time-Varying Channels, Space-time GTD.

\section{INTRODUCTION}

In previous papers, [12] and [13], we considered the design of zero-forcing decision feedback (ZF-DF) transceivers over slowly time-varying multi-input multi-output (MIMO) memoryless channels. It is assumed that instantaneous channel state information (CSI) at the transmitter (CSIT) and the receiver (CSIR) are available. The CSIT is available through feedback or time division duplex scheme [3], [13]. In [13], based on the proposed space-time generalized triangular decomposition (ST-GTD), we were able to develop space-time geometric mean decomposition (ST-GMD) ZF-DF transceiver which minimizes both the arithmetic mean square error (MSE), and the average bit error rate (BER) in high signal to noise ratio (SNR) per ST-block. The temporal precoder and the newly proposed "nested loop" receiver structure not only redistribute the MSEs in each space-time block (ST-block), but also reduce the per ST-block arithmetic MSE. However, the transceivers were mutual information lossy because of the zero-forcing constraint.

In this work, we consider the design of decision feedback transceivers without zero-forcing constraint using the same channel model. For linear time invariant (LTI) MIMO channels, the GMD-based MMSE systems [8], [9] is shown in [8] to minimize the arithmetic MSE, the geometric MSE at the feedback detector, and BER at high signal to interference plus noise ratio (SINR) region. Moreover, the design also maximizes the Gaussian mutual information. Unlike the singular value decomposition based systems which require

\footnotetext{
${ }^{1}$ This work is supported in parts by the ONR grant N00014-08-1-0709 and the California Institute of Technology.
}

bit allocation to achieve the optimal average BER [6], the geometric mean decomposition (GMD) based systems do not require bit allocation [7]. However, if the GMD-based MMSE system is directly applied to the time-varying channels, the average BER and MSE are not optimized.

In this paper, we apply ST-GTD for the design of MMSE transceiver. The proposed space-time GMD MMSE transceiver (ST-GMDM) with space-time powerloading maximizes Gaussian mutual information over the equivalent channel seen by each ST-block. Moreover, it jointly minimizes the arithmetic MSE for each ST-block and the per ST-block average BER in convex region. Hence, the average BER over the entire transmission is reduced. The details and proofs are given in [14]. Like the ST-GMD ZF transceiver in [13], the ST-GMD MMSE transceiver requires instantaneous CSIT, CSIR and channel prediction. For the applications where channel prediction is not practical, we propose the "causal" ST-GTD MMSE transceiver (CST-GTDM) which does not require channel prediction. The simulation shows the CST-GTDM has asymptotic BER performance like ST-GMD MMSE transceiver when ST-block size increases. In any case, ST-GMD MMSE transceiver serves as a performance benchmark for the general class of ST-GTD MMSE transceivers. In the convex region, which corresponds to moderate high SINR or all SINR values (depending on the modulation constellation), we show that the proposed MMSE transceivers have better BER performance than the conventional GMD-based MMSE transceiver. Moreover, the average BER of the proposed systems are non-increasing functions of ST-block size in convex region and non-decreasing functions in concave region. Simulations also demonstrate the superior BER performance of the proposed MMSE transceivers over the corresponding $\mathrm{ZF}$ transceivers. ${ }^{2}$

\section{SYSTEM MODEL AND REVIEW}

In this paper, we consider the narrowband block fading MIMO channel model [4]. The channel remains constant over the coherence period of $T_{c}$ symbol vectors and varies independently. One data block which consists of $T_{c}$ symbol vectors is transmitted during a coherence (block) interval. For simplicity of analysis, we set $T_{c}=1$. Hence, the channel model is given by

$$
\mathbf{y}(k)=\mathbf{H}(k) \mathbf{a}(k)+\mathbf{w}(k)
$$

\footnotetext{
${ }^{2}$ Notations: Upper case bold letters are reserved for matrices and lower case bold letters for vectors. $(\cdot)^{T}$ and $(\cdot)^{\dagger}$ denote the transpose and the conjugate transpose, respectively. $x_{i}$ or $[\mathbf{x}]_{i}$ denotes the $i$ th element of a vector $\mathbf{x}$; $A_{i, j}$ or $[\mathbf{A}]_{i, j}$ denotes the $(i, j)$ th element of a matrix $\mathbf{A} . \mathbf{I}_{M}$ denotes the $M \times M$ identity matrix. $E(\cdot)$ stands for expectation. $\operatorname{diag}(\mathbf{x})$ is a diagonal matrix with the entries of $\mathbf{x}$ on the diagonal. $\mathbf{u} \prec+\mathbf{v}$ and $\mathbf{u} \prec x \mathbf{v}$ denote additive and multiplicative majorization, respectively [5], [10].
} 


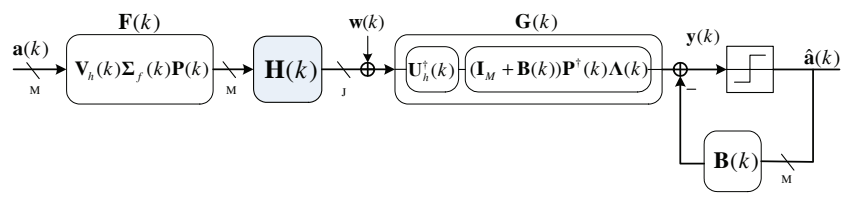

Fig. 1. The GMD-based MMSE system.

where $k$ is the coherence block index, $\mathbf{H}(k)$ is a $J \times M$ full rank channel matrix with $J \geq M$, and $\mathbf{a}(k)$ is an $M \times 1$ symbol vector from the $k$ th block with each element $[\mathbf{a}(k)]_{i}$ chosen from the alphabet $\chi$ of finite size. We assume $E\left(\mathbf{a}(k) \mathbf{a}^{\dagger}\left(k^{\prime}\right)\right)=\sigma_{a}^{2} \delta\left(k-k^{\prime}\right) \mathbf{I}_{M}$. The noise $\mathbf{w}(k)$ is a $J \times 1$ Gaussian random process vector with $E(\mathbf{w}(k))=\mathbf{0}$ and $E\left(\mathbf{w}(k) \mathbf{w}^{\dagger}\left(k^{\prime}\right)\right)=\sigma_{w}^{2} \delta\left(k-k^{\prime}\right) \mathbf{I}_{J}$. And $\mathbf{y}(k)$ is the $J \times 1$ received vector. At the $k$ th coherence interval, $\mathbf{H}(k)$ is assumed to be known to the transmitter and receiver.

Fig. 1 shows the optimal GMD MMSE transceiver, which has been shown to be optimal in average BER at high SNR and maximizes the Gaussian mutual information for LTI channels [8], [9] and [11]. Now, we apply the structure directly to the time-varying channel $\mathbf{H}(k)$ and refer to it as the conventional GMD-based MMSE transceiver. Since both the transmitter and receiver have perfect CSI at current block time $k$, the $M \times M$ precoding matrix $\mathbf{F}(k)$, the $M \times J$ feedforward matrix $\mathbf{G}(k)$, and the $M \times M$ feedback matrix $\mathbf{B}(k)$ are determined by $\mathbf{H}(k)$. The $J \times M$ matrix $\mathbf{U}_{h}(k)$ and the $M \times M$ matrix $\mathbf{V}_{h}(k)$ can be obtained from the singular value decomposition (SVD) of $\mathbf{H}(k)$,

$$
\mathbf{H}(k)=\mathbf{U}_{h}(k) \boldsymbol{\Sigma}_{h}(k) \mathbf{V}_{h}^{\dagger}(k),
$$

where $\mathbf{U}_{h}^{\dagger}(k) \mathbf{U}_{h}(k)=\mathbf{I}_{M}, \mathbf{V}_{h}^{\dagger}(k) \mathbf{V}_{h}(k)=\mathbf{I}_{M}$ and $\boldsymbol{\Sigma}_{h}(k)=$ $\operatorname{diag}\left(\sigma_{h, 0}(k), \sigma_{h, 1}(k), \ldots, \sigma_{h, M-1}(k)\right) . \sigma_{h, i}(k)$ is the $i$ th singular value of $\mathbf{H}(k)$. The power loading matrix $\boldsymbol{\Sigma}_{f}(k)$ is a diagonal matrix

$$
\boldsymbol{\Sigma}_{f}(k)=\operatorname{diag}\left(\sigma_{f, 0}(k), \sigma_{f, 1}(k), \ldots, \sigma_{f, M-1}(k)\right),
$$

where $\sigma_{f, i}^{2}(k)$ are determined by the spatial water-filling formula

$$
\sigma_{f, i}^{2}(k)=\left\{\begin{array}{cc}
\frac{1}{\lambda(k)}-\frac{\sigma_{w}^{2}}{\sigma_{a}^{2} \sigma_{h, i}^{2}(k)}, & 0 \leq i \leq L(k)-1 \\
0, & L(k) \leq i \leq M-1 .
\end{array}\right.
$$

$L(k)-1$ is the largest number such that $1 / \lambda(k)-$ $\sigma_{w}^{2} /\left(\sigma_{a}^{2} \sigma_{h, L(k)-1}^{2}(k)\right)$ is positive and

$$
\frac{1}{\lambda(k)}=\frac{1}{L(k) \sigma_{a}^{2}}\left(P_{0}+\sigma_{w}^{2} \sum_{i=0}^{L(k)-1} \frac{1}{\sigma_{h, i}^{2}(k)}\right) .
$$

To determine the $M \times M$ matrices $\mathbf{R}(k)$ and $\mathbf{P}(k)$, we first construct the $M \times M$ diagonal matrix

$$
\boldsymbol{\Theta}(k)=\left(\mathbf{I}_{M}+\frac{\sigma_{a}^{2}}{\sigma_{w}^{2}} \boldsymbol{\Sigma}_{f}^{2}(k) \boldsymbol{\Sigma}_{h}^{2}(k)\right)^{1 / 2} .
$$

$\mathbf{R}(k)$ and $\mathbf{P}(k)$ can be obtained by the GMD [7] of $\boldsymbol{\Theta}(k)$, which is

$$
\boldsymbol{\Theta}(k)=\mathbf{Q}(k) \mathbf{R}(k) \mathbf{P}^{\dagger}(k)
$$

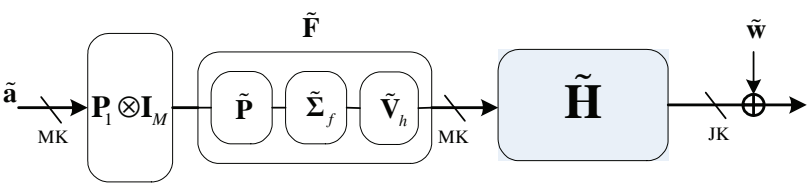

Fig. 2. The transmitter of the ST-GTD MMSE transceiver and the channel.

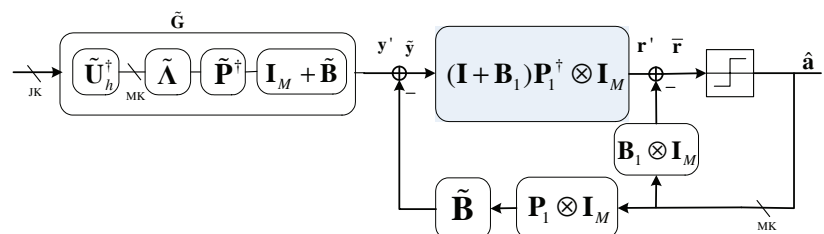

Fig. 3. The receiver of the ST-GTD MMSE transceiver.

where $\mathbf{P}(k) \in \mathbb{C}^{M \times M}$ and $\mathbf{Q}(k) \in \mathbb{C}^{J \times M}$ have orthonormal columns, and $\mathbf{R}(k) \in \mathbb{C}^{M \times M}$ is an upper triangular matrix with $\mathbf{r}(k)$ on the diagonal. The $M \times 1$ vector $\mathbf{r}(k)$ has equal elements

$$
r_{i}(k)=\sigma_{k}=\left(\prod_{i=0}^{M-1}[\Theta(k)]_{i, i}\right)^{1 / M} .
$$

Then, the $M \times M$ feedback matrix $\mathbf{B}(k)$ is given by

$$
\mathbf{B}(k)=\mathbf{D}_{\mathbf{R}}^{-\mathbf{1}}(k) \mathbf{R}(k)-\mathbf{I}_{M},
$$

where $\mathbf{D}_{\mathbf{R}}(k)=\operatorname{diag}(\mathbf{r}(k))$. The $M \times M$ diagonal matrix $\boldsymbol{\Lambda}(k)$ in $\mathbf{G}(k)$ can be obtained by

$$
\boldsymbol{\Lambda}(k)=\boldsymbol{\Sigma}_{f}(k) \boldsymbol{\Sigma}_{h}(k)\left(\boldsymbol{\Sigma}_{f}^{2}(k) \boldsymbol{\Sigma}_{h}^{2}(k)+\frac{\sigma_{q}^{2}}{\sigma_{a}^{2}} \mathbf{I}\right)^{-1} .
$$

If there is no error propagation in the DF loop, the received signal vector in front of the detector is given by

$$
\mathbf{y}(k)=\mathbf{a}(k)+\mathbf{e}(k),
$$

where

$\mathbf{e}(k)=\left(\mathbf{G}(k) \mathbf{H}(k) \mathbf{F}(k)-\mathbf{B}(k)-\mathbf{I}_{M}\right) \mathbf{a}(k)+\mathbf{G}(k) \mathbf{w}(k)$

Following the same derivation as in [8], the error covariance matrix of the error component $\mathbf{e}(k)$ can be written as

$$
\mathbf{R}_{\mathbf{e e}}=E\left(\mathbf{e e}^{\dagger}\right)=\sigma_{a}^{2} \operatorname{diag}\left(\left|r_{0}(k)\right|, \ldots,\left|r_{M-1}(k)\right|\right)^{-2} .
$$

Hence, the total MSE of the $k$ th block at the detector is

$$
\xi_{\text {gmd }}(k)=M \sigma_{a}^{2}\left(\frac{\sigma_{w}^{2} \lambda(k)}{\sigma_{a}^{2}}\right)^{\frac{L(k)}{M}}\left(\prod_{i=0}^{L(k)-1} \frac{1}{\sigma_{h, i}^{2}(k)}\right)^{1 / M} .
$$

\section{SPACE-TIME GTD MMSE TRANSCEIVERS}

In this section, we propose the ST-GTD MMSE DF transceiver based on the ST-GTD introduced in [13]. To facilitate space-time processing, $K$ blocks of symbol vectors are grouped into one space-time block as

$$
\tilde{\mathbf{a}}_{m}=\left[\begin{array}{lll}
\mathbf{a}^{T}(m K) & \ldots & \mathbf{a}^{T}(m K+K-1)
\end{array}\right]^{T},
$$

where $m$ is the ST-block index. The symbols, $m$ and $m K$, will be omitted for convenience. The equivalent MIMO channel 
matrix for the $m$ th ST-block is a $K J \times K M$ block diagonal matrix given by

$$
\tilde{\mathbf{H}}=\operatorname{diag}(\mathbf{H}(0), \mathbf{H}(1), \ldots, \mathbf{H}(K-1)) .
$$

The proposed precoder and receiver of ST-GTD transceiver are shown in Fig. 2 and Fig. 3, respectively. It is assumed that the transmitter could predict the channels $\mathbf{H}(k)$ for $0 \leq k \leq K-1$ perfectly before sending a ST-block ã and the receiver can perfectly track the channels. The decoding follows after the reception of a whole ST-block. There are well-studied methods [1], [2] which we can exploit here for the channel prediction. Even if channel prediction is impractical in some applications, the ST-GMD MMSE transceivers proposed in this section still serves as a performance benchmark for the practical transceiver proposed in next section. Since the transmitter and receiver have perfect CSI of $\tilde{\mathbf{H}}, \tilde{\mathbf{V}}_{h}$ in spatial precoder $\tilde{\mathbf{F}}$ and $\tilde{\mathbf{U}}_{h}^{\dagger}$ in feedforward matrix $\tilde{\mathbf{G}}$ can be obtained from the block SVD of $\tilde{\mathbf{H}}$ as

$$
\tilde{\mathbf{H}}=\tilde{\mathbf{U}}_{h} \tilde{\boldsymbol{\Sigma}}_{h} \tilde{\mathbf{V}}_{h}^{\dagger}
$$

where $\tilde{\mathbf{U}}_{h}, \tilde{\mathbf{V}}_{h}$ and $\tilde{\boldsymbol{\Sigma}}_{h}$ are block diagonal matrices with $\mathbf{U}_{h}(k), \mathbf{V}_{h}(k)$ and $\boldsymbol{\Sigma}_{h}(k)$ on the diagonals, respectively. $\mathbf{U}_{h}(k), \mathbf{V}_{h}(k)$ and $\boldsymbol{\Sigma}_{h}(k)$ are from the SVD of $\mathbf{H}(k)$ as in (2). Notice that $0 \leq k \leq K-1$.

ST-GTD in [13] is applied to get $\tilde{\mathbf{P}}, \mathbf{P}_{1}, \tilde{\mathbf{B}}$ and $\mathbf{B}_{1}$ for the precoder and the receiver as follows: firstly, we construct the block diagonal matrix

$$
\tilde{\boldsymbol{\Theta}}=\operatorname{diag}(\boldsymbol{\Theta}(0), \boldsymbol{\Theta}(1), \ldots, \boldsymbol{\Theta}(K-1)),
$$

where $\Theta(k)$ is given by (6). Applying the spatial GMD to $\boldsymbol{\Theta}(k)$ for all $k$, we have $\boldsymbol{\Theta}(k)=\mathbf{Q}(k) \mathbf{R}(k) \mathbf{P}^{\dagger}(k)$ as in (7). One can obtain the spatial precoder $\tilde{\mathbf{P}}$ from the block GMD of $\tilde{\Theta}$ as

$$
\tilde{\Theta}=\tilde{\mathbf{Q}} \tilde{\mathbf{R}} \tilde{\mathbf{P}}^{\dagger},
$$

where $\tilde{\mathbf{P}}, \tilde{\mathbf{R}}$ and $\tilde{\mathbf{Q}}$ are block diagonal matrix consisting of $\mathbf{P}(k), \mathbf{R}(k)$ and $\mathbf{Q}(k)$ on the diagonals, respectively. Let

$$
\mathbf{d}=\left[\sigma_{0}, \ldots, \sigma_{K-1}\right]^{T},
$$

where $\sigma_{i}$ is defined in (8). $\tilde{\mathbf{R}}$ can be factored as

$$
\tilde{\mathbf{R}}=\mathbf{D}_{\mathbf{R}}\left(\mathbf{I}_{K M}+\tilde{\mathbf{B}}\right)
$$

where $\mathbf{D}_{\mathbf{R}}=\mathbf{\Sigma} \otimes \mathbf{I}_{M}, \mathbf{\Sigma}=\operatorname{diag}(\mathbf{d})$, and the spatial feedback matrix $\tilde{\mathbf{B}}$ is a block diagonal matrix with $\mathbf{B}(k)$ on the diagonal. $\mathbf{B}(k)$ are $M \times M$ strictly upper triangular matrices given by (9).

Secondly, the temporal generalized triangular decomposition (GTD) [10], [13] of $\boldsymbol{\Sigma}$ gives the temporal precoder $\mathbf{P}_{1}$. We decompose $\boldsymbol{\Sigma}$ as

$$
\boldsymbol{\Sigma}=\mathbf{Q}_{1} \mathbf{R}_{1} \mathbf{P}_{1}^{\dagger}
$$

where $\mathbf{P}_{1}$ and $\mathbf{Q}_{1}$ are $K \times K$ unitary matrices, and $\mathbf{R}_{1}$ is a $K \times K$ upper triangular matrix. The necessary and sufficient condition for the GTD in (21) to be possible is

$$
\mathbf{r}_{1} \prec \times \mathbf{d}
$$

in which $\mathbf{r}_{1}$ is a $K \times 1$ vector consisting of diagonal elements of $\mathbf{R}_{1}$ and $\mathbf{d}$ is given by (19). The temporal domain feedback matrix $\mathbf{B}_{1}$ is given by

$$
\mathbf{B}_{1}=\mathbf{D}_{\mathbf{R}_{1}^{-1}}^{-1} \mathbf{R}_{1}-\mathbf{I}_{K}
$$

where $\mathbf{D}_{\mathbf{R}_{1}}=\operatorname{diag}\left(\mathbf{r}_{1}\right)$. So, the ST-GTD decomposes $\tilde{\boldsymbol{\Theta}}$ as $\left.\tilde{\mathbf{\Theta}}=\tilde{\mathbf{Q}}\left(\left(\mathbf{Q}_{1} \mathbf{D}_{\mathbf{R} 1}\left(\mathbf{I}_{K}+\mathbf{B}_{1}\right) \mathbf{P}_{1}^{\dagger}\right) \otimes \mathbf{I}_{M}\right)\left(\mathbf{I}_{K M}+\tilde{\mathbf{B}}\right)\right) \tilde{\mathbf{P}}^{\dagger}$,

which is entirely different from the direct GMD on $\tilde{\Theta}$. For the design of ST-GMD MMSE transceiver, the temporal GTD of $\Sigma$ in (21) is replaced by the temporal GMD in which all the entries of $\mathbf{r}_{1}$ in (22) equal $\left(\prod_{k=0}^{K-1} \sigma_{k}\right)^{1 / K}$.

\section{A. Space-time power loading}

The power loading matrix $\tilde{\boldsymbol{\Sigma}}_{f}$ in the transmitter is a $K M \times$ $K M$ block diagonal matrix

$$
\tilde{\boldsymbol{\Sigma}}_{f}=\operatorname{diag}\left(\boldsymbol{\Sigma}_{f}(0), \boldsymbol{\Sigma}_{f}(1), \ldots, \boldsymbol{\Sigma}_{f}(K-1)\right) .
$$

If we use spatial water-filling formula in (4) for $\boldsymbol{\Sigma}_{f}(k)$, the power loading matrix only uses the instantaneous CSI and allocates power on a per-block basis. Here, since $\mathbf{H}(k)$ for $0 \leq k \leq K-1$ are available by channel prediction, more sophisticated power allocation algorithm over space and time can be used. To do the water-filling over space and time, $\sigma_{f, i}^{2}(k)$ is determined by the space-time water-filling formula,

$$
\sigma_{f, i}^{2}(k)=\left\{\begin{array}{cc}
\frac{1}{\lambda}-\frac{\sigma_{w}^{2}}{\sigma_{a}^{2} \sigma_{h, i}^{2}(k)}, & 0 \leq i \leq L(k)-1 \\
0, & L(k) \leq i \leq M-1
\end{array}\right.
$$

where $L(k)-1$ is the largest number such that $1 / \lambda-$ $\sigma_{w}^{2} /\left(\sigma_{a}^{2} \sigma_{h, L(k)-1}^{2}(k)\right)$ is positive. The threshold $1 / \lambda$ is determined by the power constraint over a ST-block, i.e.,

$$
K P_{0}=\sigma_{a}^{2} \operatorname{Tr}\left(\tilde{\mathbf{F}}^{\dagger} \tilde{\mathbf{F}}\right)=\sigma_{a}^{2} \sum_{k=0}^{K-1} \sum_{i=0}^{M-1} \sigma_{f, i}^{2}(k) .
$$

Substituting (26) into (27), we have

$$
\frac{1}{\lambda}=\frac{K P_{0}+\sum_{k=0}^{K-1} \sum_{i=0}^{L(k)-1} \sigma_{w}^{2} / \sigma_{h, i}^{2}(k)}{\sigma_{a}^{2} \sum_{k=0}^{K-1} L(k)} .
$$

For each ST-block time, ã is precoded by the temporal and spatial precoders, transmitted through the channel $\tilde{\mathbf{H}}$ and pre-processed at the receiver by $\tilde{\mathbf{G}}$. The estimation of the transmitted $\tilde{\mathbf{a}}$ at the receiver is done by the successive cancellation algorithm described in Sec. III of [13].

\section{B. Mean square error at the detector}

To characterize the performance of the receiver in Fig. 3, we calculate the error covariance matrix of the noise component, $\mathbf{e}=\overline{\mathbf{r}}-\tilde{\mathbf{a}}$. Under the assumption of no error propagation in the feedback loop, the error component e can be expressed as

$$
\mathbf{e}=\mathbf{A}\left[\left(\tilde{\mathbf{G}} \tilde{\mathbf{H}} \tilde{\mathbf{F}}-\left(\tilde{\mathbf{B}}+\mathbf{I}_{M}\right)\right)\left(\mathbf{P}_{1} \otimes \mathbf{I}_{M}\right) \tilde{\mathbf{a}}+\tilde{\mathbf{G}} \tilde{\mathbf{w}}\right],
$$

where $\mathbf{A}=\left[\left(\mathbf{I}+\mathbf{B}_{1}\right) \mathbf{P}_{1}^{\dagger} \otimes \mathbf{I}_{M}\right]$. The error covariance matrix is given by

$$
\begin{aligned}
\mathbf{R}_{\mathbf{e e}} & =E\left(\mathbf{e e}^{\dagger}\right)=\sigma_{a}^{2} \mathbf{A}\left(\boldsymbol{\Sigma}^{-2} \otimes \mathbf{I}_{M}\right) \mathbf{A}^{\dagger} \\
& =\sigma_{a}^{2}\left[\mathbf{D}_{\mathbf{R}_{1}}{ }^{-1} \mathbf{D}_{\mathbf{R}_{1}}{ }^{\dagger} \otimes \mathbf{I}_{M}\right],
\end{aligned}
$$

where $\mathbf{D}_{\mathbf{R}_{1}}$ is as in (23). The second equality follows from the temporal domain GTD (21). The total MSE of the ST-GTD MMSE transceiver over a ST-block is the trace of $\mathbf{R}_{\mathbf{e e}}$,

$$
\xi_{s t-g t d m}=\sigma_{a}^{2} M \sum_{k=0}^{K-1} \frac{1}{\left|\eta_{k}\right|^{2}} \geq \sigma_{a}^{2} M K\left(\prod_{k=0}^{K-1} \frac{1}{\sigma_{k}^{2}}\right)^{\frac{1}{K}}
$$


where $\eta_{k}=\left[\mathbf{r}_{1}\right]_{k}$. The last inequality comes from AM-GM inequality and $\mathbf{r}_{1} \prec_{x} \mathbf{d}$. The lower bound on the total MSE $\xi_{s t-g t d m}$ is achieved when the ST-GMD is applied, in which we choose $\eta_{k}=\left(\prod_{i=0}^{K-1} \sigma_{i}\right)^{1 / K}$. We call this kind of ST-GTD MMSE transceiver the ST-GMD MMSE transceiver. The total mean square error of the ST-GMD MMSE transceiver is given by

$$
\xi_{s t-g m d m}=\sigma_{a}^{2} M K\left(\prod_{k=0}^{K-1} \frac{1}{\sigma_{k}^{2}}\right)^{1 / K}
$$

If space-time water filling formula in (26) is used, the minimized MSE can be shown to be

$$
\xi_{s t-g m d m}=\sigma_{a}^{2} M K\left(\frac{\sigma_{w}^{2} \lambda}{\sigma_{a}^{2}}\right)^{t}\left(\prod_{k=0}^{K-1} \prod_{i=0}^{L(k)-1} \frac{1}{\sigma_{h, i}^{2}(k)}\right)^{\frac{1}{M K}},
$$

where $t=\sum_{k=0}^{K-1} L(k) /(M K)$. This is also the smallest achievable MSE when one designs a GMD MMSE transceiver over the big equivalent channel matrix $\tilde{\mathbf{H}}$. The ST-GMD MMSE transceiver with space-time power loading is the optimal transceiver of in the class of ST-GTD MMSE transceivers in terms of total MSE. Furthermore, the error covariance matrix of ST-GMD MMSE transceiver has equal diagonal elements. Hence, for every ST-block, the ST-GMD MMSE transceiver minimizes both the arithmetic and geometric MSE, and the average un-coded BER at the high SNR region according to [8].

Although ST-GMD MMSE transceiver and the GMD MMSE transceiver designed for the big channel matrix $\tilde{\mathbf{H}}$ have the same performance, ST-GMD MMSE transceiver has lower complexity both in the operation phase and the design phase which obtains the precoder and equalizer. In the design phase, both transceivers do the same block SVD as in (16), which has complexity $O\left(K M^{3}\right)$. However, ST-GTD requires lower computational complexity, $O\left(K M^{2}+K^{2}\right)$, than the complexity of directly applying GTD on $\Theta$, which is $O\left(K^{2} M^{2}\right)$ [10], [13]. To evaluate the complexity of operations, we let the systems processes one ST-block and compare the number of multiplications and additions. The total complexity of a STGTD MMSE transceiver is $O\left(K M^{2}+K^{2} M\right)$ while that of the GMD MMSE transceiver is $O\left(M^{2} K^{2}+K M^{2}\right)$. More importantly, ST-GTD can be chosen in such a way that channel prediction is not necessary as we show next.

\section{Space-Time GTD MMSE Transceivers with FIXED TEMPORAL PRECODER}

In the previous section, the design of ST-GTD MMSE transceivers relies on the channel prediction just as the STGTD ZF transceivers in [13]. When the channel prediction becomes unavailable, we can choose $\mathbf{P}_{1}$ to be a constant DFT or Hadamard matrix just as in [13] which makes the implementation of the transmitter feasible. Now, $\mathbf{Q}_{1}$ and $\mathbf{R}_{1}$ are obtained from the QR decomposition of $\mathbf{\Sigma W}$ as

$$
\mathbf{\Sigma} \mathbf{W}=\mathbf{Q}_{1} \mathbf{R}_{1}
$$

where $\mathbf{Q}_{1}$ is also a $K \times K$ unitary matrix and $\mathbf{R}_{1}$ is a $K \times K$ upper triangular matrix. The conventional spatial water-filling formula in (4) is used to obtain power loading matrix $\boldsymbol{\Sigma}$. The detection at the receiver is exactly the same as in the ST-GTD MMSE transceiver (Fig. 3). We call this kind of transceiver the causal ST-GTD MMSE transceiver (CST-GTDM). It is in fact a subclass of ST-GTD MMSE transceiver. The error covariance matrix of the error component in front of the detector is given by (30). And the total mean square error $\xi_{c s t-g t d m}$ is given by $(31)$.

Now, we compare the performance of the conventional GMD-based MMSE system [8], the ST-GMD MMSE transceiver, and the CST-GTD MMSE transceiver. The total MSE of the GMD-based MMSE system in one ST-block is

$$
\xi_{g m d m}=\sigma_{a}^{2} M \sum_{k=0}^{K-1} \frac{1}{\sigma_{k}^{2}}
$$

The comparison is presented in Theorem 1 .

Theorem 1: The total mean square errors over one ST-block for the three transceivers are such that

$$
\xi_{s t-g m d m} \leq \xi_{s t-g t d m} \leq \xi_{g m d m} .
$$

Proof: The first inequality follows from (31). The proof of the second inequality is similar to that of Theorem 1 in [13].

The theorem shows that the class of ST-GTD MMSE transceivers has smaller total MSE per ST-block than the conventional GMD-based MMSE system. In particular, for CST-GTDM, we have

$$
\xi_{s t-g m d m} \leq \xi_{c s t-g t d m} \leq \xi_{g m d m}
$$

The temporal precoder $\mathbf{P}_{1}$ or $\mathbf{W}_{1}$, and the "nestedfeedback-loop" receiver in ST-GMD or CST-GTD MMSE transceiver redistribute the MSEs of the blocks in each STblock and also help to reduce the per ST-block arithmetic MSE. This is in contrast to the linear temporal precoder of GMD-TP in [12] which keeps the same per ST-block arithmetic MSE while equalizing the MSEs in blocks.

\section{Numerical Results}

In this section, we present the simulation results on the average BERs of the GMD-based MMSE system , ST-GMD and CST-GTD MMSE transceivers, which are denoted by $\mathcal{P}_{\text {gtdm }}, \mathcal{P}_{c s t-g m d m}$ and $\mathcal{P}_{s t-g t d m}$, respectively. The channel model and noise are the same as described in Sec. II. The modulation scheme is 16-QAM. Both transmitter and receiver have perfect CSI at current time $k$. We assume that perfect channel prediction is available only for the MMSE and zero-forcing ST-GMD transceivers. The temporal precoding matrices of the CST-GTDM are $K \times K$ DFT matrices. $N=2^{20}$ data blocks are sent through the channels for BER performance evaluation.

Example 1: The ST-block size is $K=16$. Fig. 4 shows the BER performance of the conventional GMD-based MMSE system, CST-GTDM and ST-GMD MMSE transceivers for different SNRs. For all SNRs, $\mathcal{P}_{\text {st-gmdm }}<\mathcal{P}_{\text {cst-gtdm }}$. The performance of CST-GTDM is close to ST-GMD MMSE transceiver. At the high SNR region, $\mathcal{P}_{s t-g m d m}$ and $\mathcal{P}_{c s t-g t d m}$ are smaller than $\mathcal{P}_{\text {gmdm }}$. However, at the low SNR region, $\mathcal{P}_{s t-g m d m}$ and $\mathcal{P}_{c s t-g t d m}$ are greater than $\mathcal{P}_{\text {gmd }}$. For STGMD and CST-GTD MMSE transceivers, the errors might propagate through the entire ST-block, i.e., $K$ blocks, which is much more severe than the conventional GMD-based MMSE transceiver. Hence, in the absence of error propagation 


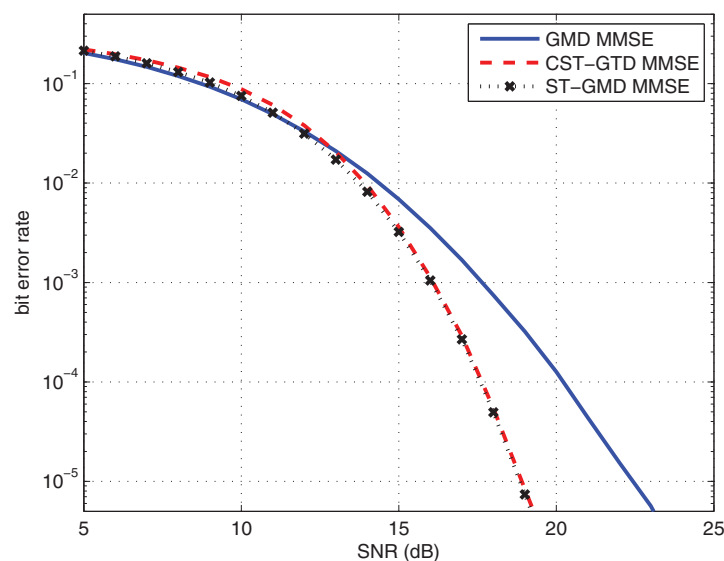

Fig. 4. BER performance of GMD, ST-GMD and CST-GTD MMSE transceivers with ST-block size $K=16$.

which corresponds to high SNR region, the ST-GMD MMSE transceiver has the best BER performance. At BER $10^{-4}$, the SNR gains of the ST-GMD and the CST-GTD MMSE transceivers over the GMD-based MMSE system are $2.7 \mathrm{~dB}$ and $2.59 \mathrm{~dB}$, respectively. Various choices of ST-block size are also considered. At the high SNR region, $\mathcal{P}_{s t-g m d m}$ and $\mathcal{P}_{\text {cst-gtdm }}$ decrease with respect to $K$ which is best illustrated by Fig. 5. Notice that the BER performance of the CST-GTD and ST-GMD MMSE transceiver are almost the same when $K=32$.

Example 2: In this example, we compare the average BER performance of the proposed ST-GMD and CST-GMD MMSE transceivers with the corresponding zero-forcing transceivers in [13]. The ST-block size $K$ is 16 . The results are shown in Fig. 6. At BER $10^{-4}$, the SNR gains of ST-GMD, and CSTGMD MMSE transceivers over their zero-forcing counterparts are $0.38 \mathrm{~dB}$ and $0.6 \mathrm{~dB}$, respectively. At BER $10^{-1}$ which corresponds to low SNR region, the gains are $1 \mathrm{~dB}$ and 1.3 $\mathrm{dB}$.

\section{CONCLUSIONS}

Two MIMO MMSE decision feedback transceivers for narrowband MIMO time-varying channels were proposed here. With perfect channel prediction, the ST-GMD MMSE transceiver is shown to be the optimal in terms of arithmetic MSE and average BER in convex region. The CST-GTDM has the same asymptotic BER performance as the ST-GMD MMSE transceiver, and has smaller arithmetic MSE than the conventional GMD-based MMSE systems. The dependency of average BER on the ST-block size has also been observed. We also see the advantage of ST-GMD MMSE transceiver over ST-GMD ZF transceiver in average BER.

\section{REFERENCES}

[1] A. Duel-Hallen, S. Hu, and H. Hallen, "Long-range prediction of fading signals," IEEE Signal Precessiong Mag., vol. 17, pp.62-75, May 2000.

[2] S. Zhou and G. B. Giannakis, "How accurate channel prediction needs to be for transmit-beamforming with adaptive modulation over Rayleigh MIMO channels?," IEEE Trans. Wireless Commun., vol. 3, no. 3, pp.12851294, July 2004.

[3] G. Lebrun, J. Gao, and M. Faulkner, "MIMO transmission over a timevarying channel using SVD," IEEE Trans. Wireless Commun., vol. 4, no. 2, pp.757-764, Mar. 2005.

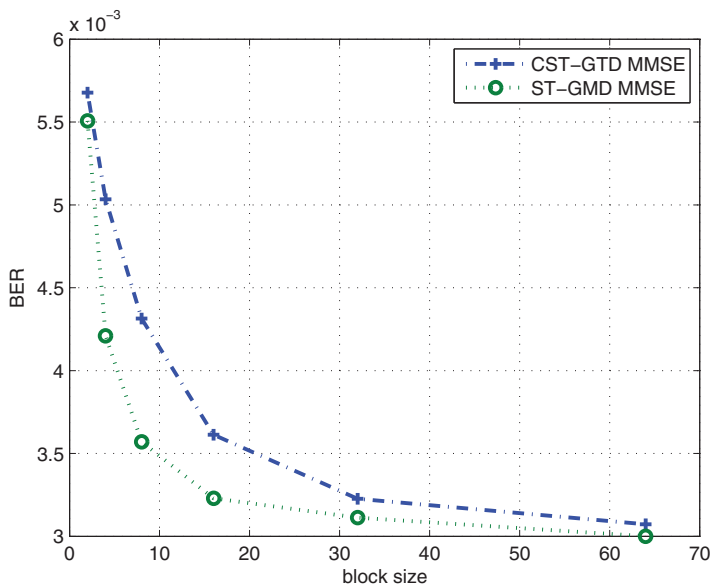

Fig. 5. BER performance v.s. block size at $\mathrm{SNR}=15 \mathrm{~dB}$.

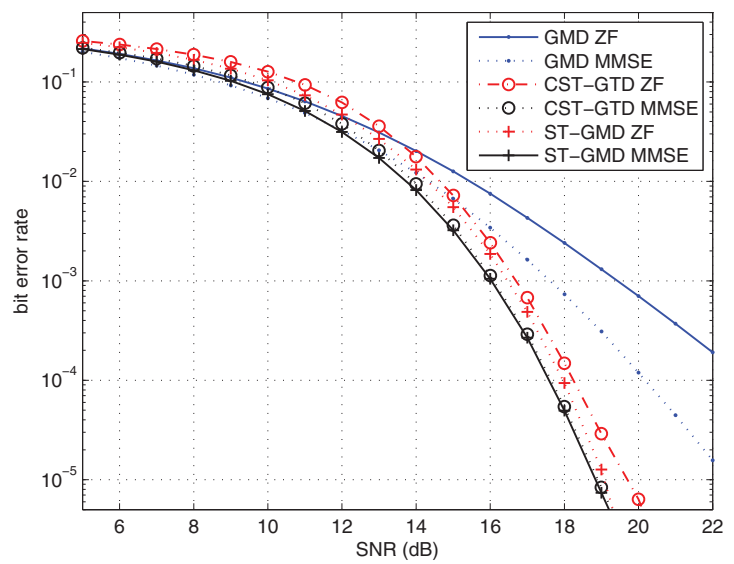

Fig. 6. BER performance of MMSE and ZF transceivers.

[4] D. Tse and P. Viswanath, Fundamentals of Wireless Communication, Cambridge Univ. Press, 2005.

[5] A. W. Marshall, and I. Olkin, Inequalities: Theory of majorization and its applications, Academic Press, 1979.

[6] G. G. Raleigh and J. M. Cioffi, "Spatio-temporal coding for wireless communication," IEEE Trans. Commun., vol.46, no.3, pp.357-366, Mar. 1998.

[7] Y. Jiang, J. Li, and W. W. Hager, "Joint transceiver design for MIMO communications using geometric mean decomposition," IEEE Trans. Sig. Proc., pp.3791-3803, Oct. 2005.

[8] F. Xu, T. N. Davidson, J. K. Zhang and K. M. Wong, "Design of block transceivers with decision feedback detection," IEEE Trans. Sig. Proc., vol. 54, no. 3, pp. 965-978, Mar. 2006.

[9] Y. Jiang, J. Li, and W. W. Hager, "Uniform channel decomposition for MIMO communications," IEEE Trans. Sig. Proc., pp.4283-4294, Nov. 2005 .

[10] Y. Jiang, W. W. Hager, and J. Li, "Generalized triangular decomposition," Mathematics of computation., Oct. 2007.

[11] P. P. Vaidyanathan, S. M. Phoong and Y. P. Lin, Signal processing and optimization for transceiver systems, Cambridge Univ. Press, 2010.

[12] C. H. Liu and P. P. Vaidyanathan, "ZF-DFE transceiver for time-varying MIMO channels with channel-independent temporal precoder," Proc. ISCAS, 2010.

[13] C. H. Liu and P. P. Vaidyanathan, "Zero-forcing DFE transceiver design over slowly time-varying MIMO channels using ST-GTD," submitted to IEEE Trans. Sig. Proc.

[14] C. H. Liu and P. P. Vaidyanathan, "MMSE decision feedback transceiver design over slowly time-varying MIMO channels using ST-GTD," in preparation. 